\title{
Uma análise da pobreza multidimensional do Nordeste metropolitano com uso de modelo de equações estruturais
}

\author{
Lucilena Ferraz Castanheira Corrêa* \\ João Policarpo Rodrigues Lima** \\ Luís Henrique Romani de Campos ${ }^{\star \star *}$
}

\begin{abstract}
A pobreza é um fenômeno social altamente complexo e a problemática desse estágio de privação é constituída pela agregação de vários fatores sociais. Diante dessa perspectiva, quanto mais informação for sendo incorporada aos estudos que envolvem esse estágio de privação, mais preciso será o diagnóstico dessa realidade. Este artigo utiliza como instrumento analítico o método de Modelagem de Equações Estruturais (MEE), empregando microdados da Pesquisa Nacional por Amostra de Domicílios (PNAD) de 2009, com foco nas regiões metropolitanas do Nordeste: Fortaleza, Recife e Salvador. Foram definidas quatro dimensões da pobreza (capacidade, bem-estar econômico, inclusão econômica e inclusão por meio das condições de moradia), em que é efetuada análise de correlação. No segundo momento, essas dimensões passam a ser analisadas sob a ótica dos seus impactos sobre a pobreza. Os resultados desse estudo reforçam a existência multidimensional das características desse estado de privação social e econômica já apontada por outros trabalhos a partir de uma modelagem mais adequada ao arcabouço teórico.
\end{abstract}

Palavras-chave: Pobreza. Bem-estar econômico. Capacidade. Inclusão econômica. Inclusão por meio das condições de moradia.

\footnotetext{
*Universidade Federal de Pernambuco - UFPE, Recife-PE, Brasil (lucilena_castanheira@hotmail.com).

** Universidade Federal de Pernambuco, Recife-PE, Brasil (jprlima@ufpe.br).

${ }^{\star \star *}$ Fundação Joaquim Nabuco, Recife-PE, Brasil (luis.campos@fundaj.gov.br).
} 


\section{Introdução}

A pobreza é a face mais dramática da concentração de renda de um país, pois se desenvolve por meio da construção e consolidação de estruturas e processos que the atribuem forma concreta de estágio de privação tanto social como econômica. Nas últimas décadas, várias abordagens conceituais foram propostas buscando instrumentos metodológicos que contribuíssem com estudos focalizados no fenômeno da pobreza. Uma importante referência nesses estudos é Sen (1999), que aborda a questão da pobreza avançando para além do corte da renda (material). Seu posicionamento é favorável ao entendimento da pobreza como um problema complexo que deve ser visto por meio das privações enfrentadas em todas as estruturas que compõem a vida das pessoas.

Os estudos que se propõem a mensurar a pobreza a partir de uma estrutura interdependente entre fatores sociais e econômicos sugerem uma abordagem multidimensional, que considere as condições de vida de um indivíduo (ou unidade domiciliar) para além da renda monetária disponível, tais como educação, saúde, acesso ao mercado de trabalho, condições de moradia, etc. Além de incluírem tais dimensões, as propostas thes conferem um contorno estrutural, ou seja, reconhecem nelas um inter-relacionamento, o que obriga as metodologias da análise da pobreza a tratarem de sistemas complexos (WAGLE, 2008; KAGEYAMA; HOFFMANN, 2006; CODES, 2005; LISTER, 2004; SEN, 2000, RAVALLION, 1996).

Para conceituar o fenômeno da pobreza sob a ótica multidimensional e estudar a pobreza em três regiões metropolitanas do Nordeste brasileiro - Região Metropolitana de Fortaleza (RMF), Região Metropolitana do Recife (RMR) e Região Metropolitana de Salvador (RMS) -, este artigo faz uso da abordagem empregada por Wagle (2008), Multidimensional poverty measurement: concepts and applications. No Brasil, a realidade da pobreza está atrelada a uma herança de injustiça social que vem desde sua colonização, eclodindo num cenário de grandes desigualdades e permanecendo um desafio histórico a ser enfrentado. Ao lado da pobreza também existe reconhecido problema de desigualdades regionais, com a porção Norte/Nordeste do país apresentando indicadores sociais bem inferiores. Portanto, a análise das regiões metropolitanas do Nordeste, evidenciando suas semelhanças e complexidades, pode contribuir para o debate acerca da construção de políticas públicas mais eficazes visando a redução deste indesejável fenômeno social.

A operacionalização da abordagem multidimensional, que é composta por uma estrutura de natureza relacional de um objeto social, utiliza a abordagem da Modelagem de Equações Estruturais (MEE) por se tratar de um método quantitativo capaz de determinar os fatores mais influentes nas condições de vida das pessoas, mostrando-se, também, apto a valorar a intensidade de tais influências, tanto direta como indiretamente (WAGLE, 2008; CODES, 2005).

Neste estudo, seguindo a abordagem defendida por Wagle (2008), ressalta-se a relevância de trabalhos que contribuam para um olhar mais aprofundado sobre o fenômeno da pobreza por meio de uma lente multidimensional. A seguir apresenta-se uma revisão de literatura 
centrada na discussão sobre a abordagem multidimensional da pobreza e nas dimensões definidas como vetores da pobreza. Posteriormente, é abordada a discussão metodológica, referente ao método quantitativo - modelos de equações estruturais -, como instrumento de análise e medição da pobreza nas regiões metropolitanas do Nordeste, além da construção dos modelos propostos utilizando a Pesquisa Nacional por Amostra de Domicílios (PNAD) 2009 , tendo como população-alvo as pessoas responsáveis pelos domicílios residentes nas regiões estudadas. Na sequência, de posse dos dados, busca-se analisar os resultados gerados a partir dos dados empíricos, utilizando a Modelagem de Equações Estruturais (MEE). Para concluir, são tecidas as considerações finais como forma de se justificar a metodologia de MEE como método plausível para estudar o fenômeno da pobreza.

\section{Vetores da pobreza: breve discussão sobre a dimensão qualitativa desse fenômeno}

Tratar a pobreza como um fenômeno multifacetado é entendê-la como resultante de um complexo e intrincado sistema de forças que se inter-relacionam e provocam o surgimento e manutenção de camadas sociais que padecem com diversos tipos de privações. Qualquer construção teórica que se pretenda viável de ser testada carece de algum esquema simplificador. Este artigo propõe um esquema a partir de quatro vetores de análise: bem-estar econômico; capacidade; inclusão econômica via mercado de trabalho; e inclusão por meio das condições de moradia. Estes vetores também podem ser entendidos como dimensões, pois em cada um deles existe um conjunto de atributos, ações e costumes sociais que os caracteriza.

Para Wagle (2008), a pobreza possui caráter multidimensional que vai muito além da renda monetária que implica ter ou não mais recursos materiais demandados em determinado estilo de vida. Ravallion (1996) defende que a busca por uma abordagem "verdadeiramente" multidimensional por meio da agregação de diversas variáveis dependentes tente resultar em algumas inter-relações complexas entre essas variáveis, estabelecendo uma base empírica credível para o combate à pobreza. Corroborando a relevância da abordagem sob a ótica multidimensional, Diallo (2010) menciona a grande contribuição dessa metodologia para melhor caracterização do público-alvo, os pobres. Diante dessa perspectiva, pode-se verificar que a pobreza é cristalizada como um fenômeno multidimensional e, para ser identificada sob essa ótica, devem ser consideradas as diversas dimensões que compõem essa realidade de privação (BARROS; CARVALHO; FRANCO, 2006).

Sumner (2004) argumenta sobre a necessidade de estudos mais complexos, que vão além da análise monetária, pelo fato de proporcionarem um melhor entendimento sobre a dinâmica do fenômeno da pobreza. Alkire (2015), por exemplo, chama a atenção para algumas variáveis presentes em estudos que utilizam as dimensões educação, saúde, habitação, entre outras, nas abordagens multidimensionais, constituindo-se em importantes instrumentos de políticas públicas na busca pela redução da pobreza. Assim, reforça-se a importância de estudos que levem em conta as características multidimensionais da 
pobreza. Wagle (2008) menciona que Chakravarty (1983) e Tsui $(1999,2002)$ foram dois pioneiros na utilização de variáveis reais - métodos quantitativos - para abordagem das características multidimensionais da pobreza. Deutsch e Silber (2005), utilizando Banco de Dados de Israel de 1995, trabalharam quatro diferentes abordagens multidimensionais ${ }^{1} \mathrm{e}$ concluíram que havia uma forte tendência positiva no uso desses índices de pobreza multidimensional para identificar o fenômeno da pobreza com maior grau de confiabilidade. Betti, D’Agostino e Neri (2002) empregaram a abordagem multidimensional para identificar a dinâmica da pobreza e como os fatores sociodemográficos a influenciam. Kageyama e Hoffmann (2006), por exemplo, produziram um estudo da pobreza multidimensional para o Brasil usando, além da renda, o acesso aos três serviços básicos no que tange à salubridade de moradia: água encanada; instalação sanitária; e iluminação elétrica. Barros, Carvalho e Franco (2003) construíram, a partir da abordagem multidimensional, o Índice de Desenvolvimento da Família - IDF, formatando-o sobre seis dimensões, 26 componentes e 48 indicadores. Codes (2005) defende a utilização de um método quantitativo que contribua para a explicação da natureza relacional de um objeto social tão complexo como o fenômeno da pobreza. 0 objeto central dessa seção consiste no levantamento de um embasamento teórico para justificar o uso da metodologia proposta por Wagle (2008), com o objetivo de medir e avaliar a pobreza a partir das relações mútuas entre as dimensões, por meio de uma abordagem multidimensional.

O bem-estar econômico, usado com certa frequência na literatura, é extraído da valorização das relações humanas, sociais ou econômicas e, por longo tempo, foi o cerne de estudos sobre a pobreza. Uma das formas de se observar o bem-estar é por meio da dimensão econômica, seja pela insuficiência de recursos monetários, seja pela baixa capacidade de consumo das pessoas (ou famílias) (BARROS; MENDONÇA, 1995; RAMOS; REIS, 1995; ROCHA, 1997; ORSHANSKY, 1965). Nesse sentido, segundo Wagle (2008), o bem-estar econômico tem como premissa captar os recursos físicos que determinam a qualidade de vida material. Para Ringen (1987), por exemplo, a pobreza pode ser definida e mensurada tanto direta (consumo) como indiretamente (renda). Nessa perspectiva, o bem-estar passa a ser um indicador do baixo nível de vida das pessoas, via inadequação dos recursos materiais. Assim, bem-estar econômico pode ser entendido sob a ótica de que a renda e o consumo de bens e serviços (por exemplo, habitação, transporte, vestuário) são a materialização da distribuição dos recursos disponíveis, o que significa, então, que tais variáveis se mostram confiáveis para se apresentarem como instrumentos de análise para diagnosticar o fenômeno da pobreza (ROCHA, 1997, 1998, 2005; RAMOS; REIS, 1995).

Cumpre destacar que a tradição mais prolífica de estudos sobre a pobreza entende que o bem-estar econômico é suficiente para mensurar em que medida uma pessoa (ou família) é pobre. Entretanto, olhar apenas esta dimensão leva à tentativa de construção de “linhas de pobreza”, que são muito úteis para quantificação de efetividades de políticas

\footnotetext{
${ }^{1}$ O objetivo desse trabalho não foi comparar as diferentes abordagens multidimensionais, mas sim apenas indicar algumas delas: conjuntos fuzzy; teoria da informação; análise de eficiência; e derivação dos axiomas de índices de pobreza.
} 
públicas, mas pouco confiáveis para realmente auxiliar em como as políticas públicas devem ser desenhadas. Por trás do argumento de que toda a questão da pobreza pode se resumir na renda subjaz a ideia utilitarista de que o bem-estar pode ser medido pelo nível de consumo que uma pessoa (ou família) pode efetivar. Citro e Michel (1995) alertam para o fato de que esse instrumental de análise para medir a extensão da pobreza carece de ser revisto tanto entre os grupos populacionais quanto em áreas geográficas do país, pois é necessária a busca por uma imagem mais real desse fenômeno ao longo do tempo.

A partir dessa perspectiva, o não atendimento das capacidades básicas passa a ser muito mais perverso sob o prisma de liberdade e justiça do que as definições na literatura que consideram a pobreza limitação de determinada quantidade de recursos monetários ${ }^{2}$ (SEN, 1999, 2000, 2011; ALKIRE, 2015). Para Sen (2011), os mais pobres entre os pobres são aqueles indivíduos que vivenciam a carência de capacidade além da renda, pois tendem a sofrer o chamado efeito "desvantagem da conversão", ${ }^{3}$ ou seja, a inaptidão os leva a não conseguir obter uma boa qualidade de vida mesmo com acesso a renda e consumo. Essa "desvantagem da conversão" só será superada por meio de esforços que levem em conta o caráter multidimensional da pobreza, centrando a devida importância nos conjuntos de recursos básicos, incluindo educação, saúde, nutrição, etc., que se apresentam como pilares fundamentais para a redução real da pobreza (ALKIRE, 2015; WAGLE, 2008; SEN, 2000). Nesse sentido, Bourguignon e Chakravarty (2003) desenvolveram um estudo utilizando a abordagem multidimensional para o Brasil e constataram uma forte correlação entre renda e educação para identificar o fenômeno da pobreza. Diallo (2010), a partir de um modelo multidimensional, verificou que $54,48 \%$ dos domicílios, na Guinea, são estruturalmente pobres e o baixo nível de escolaridade está entre as dimensões que mais indicam o grau de privação dessas famílias.

A inclusão social pode ser considerada mais um pilar na estrutura pela busca da redução da pobreza. Diante dessa perspectiva, Marió (2005) menciona que, nas sociedades modernas, o mercado de trabalho passou a ser um dos principais mecanismos para efetivar a inclusão social de uma parcela significativa da população mundial. Singer (1996) identificou que no Brasil fatores estuturais como desigualdade educacional, emprego informal e trajetória crescente nas taxas de empregos estão inter-relacionados com a exclusão social no país. Nesse sentido, o acesso ao mercado de trabalho passou a ser um fator de estudo para explicar a exclusão social em muitos estudos acadêmicos (WAGLE, 2008; BURCHARDT et al., 2002; OPEL, 2000). Dessa forma, a inclusão da parcela menos favorecida da sociedade enfrenta constantes desafios para superar as deficiências do meio social, lutando árdua e continuamente pela conquista de oportunidades no mercado de trabalho. As maneiras

\footnotetext{
${ }^{2}$ Essa metodologia determina um limite de renda e, caso o indivíduo esteja abaixo desse limite, ele é considerado pobre.

${ }^{3}$ Sen (2011, p. 293) cita, como exemplo de "desvantagem de conversão", dados empíricos sobre a pobreza no Reino Unido levantados por Wiebke Kuklys (2005), mostrando que 17,9\% dos indivíduos viviam com renda abaixo da linha de pobreza. Essa proporção passa para $23,1 \%$ quando se constata que existe pelo menos um membro incapacitado na família. Logo, segundo o autor, esse hiato de cinco pontos percentuais está diretamente relacionado à deficiência de renda, resultado da privação de capacidades.
} 
como o capital é utilizado e remunerado nas atividades econômicas do país acarretam forte impacto no nível de pobreza de grande parcela da população. Nesse contexto, o mercado de trabalho desempenha papel predominante na inclusão social desses atores sociais.

A exclusão social também pode ser analisada sob a ótica da precariedade das habitações. 0 dimensionamento da carência de muitos domicílios brasileiros evidencia uma segregação socioespacial que leva várias famílias de baixa renda a viverem em áreas desestruturadas e com a aprovação do poder público (BARBOSA; GAVIOLI; YAMANISHI, 2003). Barros, Carvalho e Franco (2006), na elaboração do Índice de Pobreza Familiar (IPF), construído por meio de seis dimensões, verificaram que a dimensão carência habitacional ${ }^{4}$ tinha forte correlação com as dimensões acesso ao conhecimento, acesso ao trabalho, escassez de recursos e desenvolvimento infantil. No Brasil, especificamente, ainda existem milhões de famílias que vivem em moradias sob condições inadequadas. Nessa área, o setor público é o responsável pela infraestrutura ao redor dessas moradias, em termos de saneamento básico, coleta de lixo, procedência da água disponibilizada, segurança, etc. No entanto, a precária situação das condições sociais de muitas famílias no país pode, muitas vezes, ser verificada a partir das condições de habitação que refletem na saúde desses membros da sociedade (NERY, 2004; BARBOSA; GAVIOLI; YAMANISHI, 2003; BARRETO et al., 2011; HOLANDA et al., 2003; RODRIGUES, 2005).

0 caráter multidimensional do fenômeno da pobreza dividido pelas quatro dimensões apresentadas nessa seção - bem-estar econômico, capacidade, inclusão econômica e inclusão por meio das condições de moradias - mostra aspectos estritamente inter-relacionados tanto pelo grau quanto pela intensidade com o fenômeno da pobreza. Diante de tal evidência, este artigo promove uma explicação de natureza relacional, ou seja, como a pobreza pode estar relacionada com esses vetores, por meio de uma abordagem quantitativa denominada de Modelagem de Equações Estruturais (MEE) (CODES, 2005).

\section{Metodologia}

Tratar a pobreza a partir de distintas dimensões significa enfrentar um desafio metodológico. Isso porque as dimensões são, na verdade, construções teóricas, ou seja, não existem mensurações únicas ou viáveis às mesmas. A alternativa proposta neste artigo é abordar tais aspectos utilizando a metodologia da Modelagem de Equações Estruturais (MEE), que objetiva a análise da relação entre as variáveis observadas ${ }^{5}$ (contínuas, ordinais, dicotômicas ou censuradas) e as variáveis latentes - dimensões (variáveis não observadas), como já abordado por outros autores (CODES, 2005).

\footnotetext{
${ }^{4}$ Essa dimensão engloba: propriedade do imóvel; déficit habitacional; capacidade de abrigar; acesso inadequado a água; acesso inadequado a esgotamento sanitário; falta de acesso a coleta de lixo; falta de acesso a eletricidade; e falta de acesso a bens duráveis

${ }^{5}$ Conhecidas também como variáveis independentes, covariáveis, variáveis preditivas. Os valores das variáveis exógenas são assumidos como dados, ou seja, o modelo não tenta explicá-los (CODES, 2008, p. 31).
} 
A MEE é dividida em fases, conforme defendido por Hair et al. (2009) e identificadas por Lamare (2002) e Silva (2006). Estas fases são: desenvolver um modelo teórico com a justificativa sobre as escolhas das variáveis com embasamento teórico; demonstrar, a partir da representação gráfica, o caminho dessas relações causais; converter o diagrama de caminho em modelo de mensuração (em que as variáveis latentes - dimensões - são regredidas em relação às variáveis mensuráveis - measurement model), resultando em construtos e o modelo estrutural (structural model) em que se aplica a técnica de regressão múltipla, ou seja, analisa-se a inter-relação entre variáveis latentes; e avaliar as estimativas do modelo e qualidade de ajuste. Os procedimentos de ajuste dos modelos estão centrados na verificação, correção e confirmação do modelo proposto. Deve-se ressaltar que a MEE é baseada em relação de causa e efeito, ou seja, mesmo que não se conheça uma medida da causa, conhecem-se medidas (e elas estão disponíveis) dos efeitos. Assim, a técnica parte da variabilidade das variáveis de efeito e da estrutura causa-efeito para construir mensurações factíveis para as causas.

Neste artigo, o comportamento relacional entre as variáveis que identificam a pobreza é tratado com o uso da técnica estatística da Análise Fatorial Confirmatória (AFC), ou seja, quando os relacionamentos obtidos são predeterminados e, a partir daí, ocorre a confirmação ou rejeição das conexões levantadas como hipótese do modelo proposto diante de determinada teoria. Para a confirmação do embasamento teórico (descrito na seção anterior) sobre a abordagem multidimensional da pobreza, o modelo proposto desse estudo é repesentado pelo Diagrama de Caminhos (Figura 1), que mostra a pobreza e suas dimensões como variáveis latentes - por isso, marcadas com elipses - enquanto as variáveis que podem ser medidas (observadas) estão marcadas por retângulos. Alterações nos níveis de pobreza afetam todas as dimensões, que, por sua vez, afetam as variáveis que podem ser medidas, o que é representado pelas setas. Diante dessa perspectiva, é importante identificar o grau de correlação que a dimensão superior - pobreza - possui com os construtos (dimensões) de primeira ordem: bem-estar econômico (Bee); capacidade (Capac); inclusão econômica (le); e inclusão por meio das condições de moradia (Icm). Os elementos $\zeta_{i}$ são os erros de mensuração de Bee, Capac, le e Icm.

Formalmente, o modelo multidimensional proposto pode ser representado pelas equações descritas a seguir. A equação 1 representa as variáveis latentes - dimensões - de bem-estar econômico $\left(\eta_{1}\right)$, capacidade $\left(\eta_{2}\right)$, inclusão econômica $\left(\eta_{3}\right)$ e inclusão por meio das condições de moradia $\left(\eta_{4}\right)$, que são determinadas pelas variáveis observadas (y). A equação 2 determina as relações entre os fatores de primeira e segunda ordens - a pobreza ( () . 0 termo $\mathrm{B} \eta$ da equação 2 deve ser desconsiderado quando existem somente fatores de segunda ordem e nenhum dos fatores de primeira ordem causa efeitos diretos sobre os de segunda ordem.

$y=\Lambda_{\mathrm{y}} \eta+\varepsilon$

$\eta=\mathrm{B} \eta+\Gamma \xi+\zeta$ 
Assim, o modelo de segunda ordem passa a ser representado conforme a equação 3.

$\eta=\Gamma \xi+\zeta$

Em resumo, a equação geral do modelo $\mathrm{AFC}$ de segunda ordem deriva de uma combinação entre a equação 3 (representando o modelo estrutural de ordem mais elevada) e a equação 1 (representando o modelo de primeira ordem, modelo de medição). A decomposição pode se apresentar da seguinte maneira, segundo Byrne (1998):

$y=\Lambda_{y}(\Gamma \xi+\zeta)+\varepsilon$

FIGURA 1

Diagrama de Caminho do Modelo de análise fatorial do modelo proposto
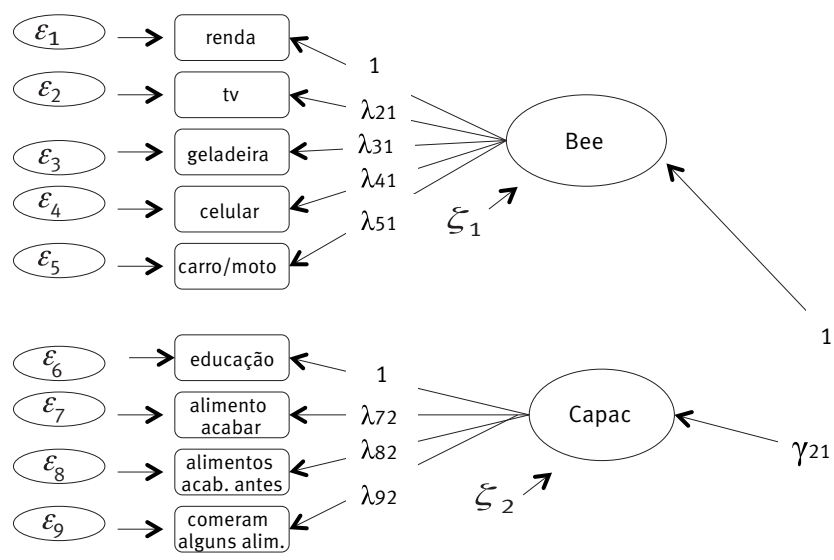

1

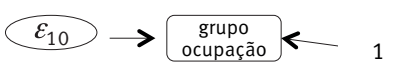

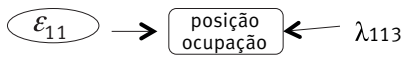

$\varepsilon_{12} \rightarrow \lambda_{123}$
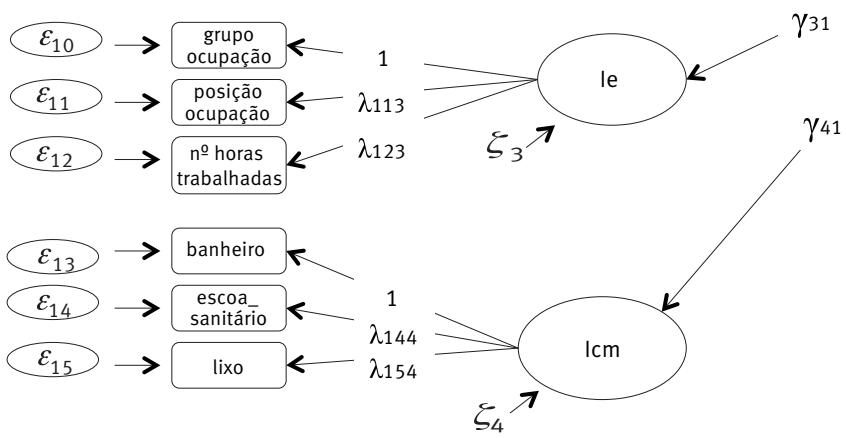

Nota: As variáveis observadas utilizadas no modelo (retângulos) estão descritas na seção de dados e variáveis.

É importante ressaltar que a MEE tem como premissa básica assumir distribuição normal das suas variáveis. Para isso, Muthén e Muthén (2007) indicam utilizar o estimador WLSMV - estimador robusto ponderado - para corrigir a anormalidade da distribuição quando são empregadas variáveis categóricas (ou seja, dicotômicas ou politômicas) que venham compor os construtos na MEE. Vale salientar que o presente estudo fará uso do método WLSMV (Weighted Least Square Means and Variance Adjusted) para suas análises. Para realizar a estimativa sugerida, optou-se pelo pacote estatístico Mplus, que é capaz 
de lidar com o MLSMV em ambientes de amostras complexas, como a Pesquisa Nacional por Amostra de Domicílios (PNAD).

Vale destacar que, quando se trabalha com pesquisas amostrais complexas, como a PNAD, é preciso considerar os pesos das unidades amostrais (aqui, as pessoas) e as informações sobre desenho amostral, a fim de alcançar estimativas não viciadas dos parâmetros. Os pesos amostrais ajustados são informados nos microdados da PNAD. ${ }^{6}$ No caso do arquivo de pessoas, a variável de peso amostral é V4729 (peso), utilizada na análise. ${ }^{7}$ É importante ressaltar, também, que o software Mplus tende a oferecer as melhores opções para a modelagem de AFC com dados categóricos. Isso é devido, em parte, ao estimador WLSMV (mínimos quadrados ponderados robustos ajustados pela média e variância), que atualmente se encontra disponível apenas nesse programa (BROWN, 2006).

\section{Variáveis de estudo}

Os dados empíricos que dão sustentação aos modelos de equações estruturais propostos baseiam-se na Pesquisa Nacional por Amostra de Domicílios (PNAD) de 2009. 0 subconjunto da amostra utilizada foi montado a partir da junção dos dados dos responsáveis pelos domicílios (arquivo de pessoas) ${ }^{8}$ com dados dos domicílios. Foi feito corte para pessoas residentes nas três regiões metropolitanas estudadas: de Fortaleza, de Recife e de Salvador.

É importante mencionar a função de algumas variáveis observadas no modelo proposto, de acordo com a Figura 1. As variáveis TV em cores, geladeira e telefone móvel (celular) são utilizadas como proxies do consumo. A variável carro ou motocicleta para uso pessoal se apresenta como proxy do acesso ao crédito, ${ }^{9}$ no entanto, outras formas de medir acesso ao crédito poderiam ser previstas em outras pesquisas domiciliares. As variáveis do módulo para estimação da insegurança alimentar no domicílio foram obtidas a partir das perguntas "se os moradores tiveram a preocupação com o fato de que os alimentos acabassem antes de poderem comprar ou receber mais comida", "se os alimentos acabaram antes que os moradores deste domicílio tivessem dinheiro para comprar mais comida" e "se os

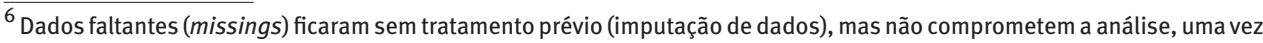
que o software Mplus oferece uma opção para lidar com dados faltantes. Para mais informações, ver Muthén e Muthén (2007).

${ }^{7}$ Para detalhes de como o Mplus lida com amostras complexas, ver Asparouhov e Muthén (2010). Basicamente informamse o peso e dados do desenho amostral, que estão disponíveis na documentação da PNAD. Os autores descrevem que o procedimento é suficiente para resolver a maioria dos problemas de estimação de modelos para situações em que o desenho amostral complexo extrapola a intenção inicial. 0 software permite uso de procedimentos como bootstrap e jackknife para resolver eventuais problemas de mal entendimento do desenho amostral original. Neste artigo não foram utilizados estes procedimentos.

${ }^{8} 0$ uso de informações dos responsáveis pelo domicílio pressupõe que as informações socioeconômicas desses são boas aproximações das condições de todos os moradores do domicílio.

${ }^{9}$ Vale ressaltar que não se deve afirmar que essa seja uma boa proxy, mas foi o possível nesta base de dados.
} 
moradores deste domicílio comeram apenas alguns alimentos que ainda tinham porque o dinheiro acabou". ${ }^{10}$

Nessa perspectiva, as escalas foram construídas de acordo com a proposta de Codes (2005) em seu estudo para Salvador. ${ }^{11}$ As variáveis e suas respectivas categorias utilizadas neste trabalho se encontram dispostas no Quadro 1. A análise dessas relações mediante o Diagrama de Caminho (Figura 1) apresenta o modelo hipotético em duas ordens e é realizada em várias etapas, conforme exposição na seção subsequente.

\section{QUADRO 1}

Variáveis latentes e observáveis utilizadas, em nível domiciliar, no Modelo de Análise Confirmatória (AFC)

\begin{tabular}{|c|c|}
\hline Variáveis latentes & Variáveis observáveis \\
\hline $\begin{array}{l}\text { Bem-estar econômico } \\
\text { (Bee) }\end{array}$ & $\begin{array}{l}\text { In da renda domiciliar per capita mensal de todas as fontes de rendimento (R\$) } \\
\text { Domicílio possui TV a cores }(1=\text { não; } 2=\operatorname{sim}) \\
\text { Domicílio possui geladeira (1=não; } 2=\operatorname{sim}) \\
\text { Possui telefone móvel } \\
\text { (1=não; } 2=\operatorname{sim)~} \\
\text { Domicílio possui carro ou motocicleta de uso pessoal (1=não; } 2=\text { carro; } \\
\text { 3=motocicleta; } 4=\text { carro e motocicleta) }\end{array}$ \\
\hline Capacidade (Capac) & $\begin{array}{l}\text { Anos de estudo completos do responsável pelo domicílio } \\
\text { Nos últimos três meses, os moradores tiveram a preocupação com o fato de que } \\
\text { os alimentos acabassem antes de poderem comprar ou receber mais comida } \\
\text { (1=não; } 2=\operatorname{sim}) \\
\text { Nos últimos três meses, os alimentos acabaram antes que os moradores desse } \\
\text { domicílio tivessem dinheiro para comprar mais comida(1=não; } 2=\text { sim) } \\
\text { Nos últimos três meses, os moradores deste domicílio comeram apenas alguns } \\
\text { alimentos que ainda tinham porque o dinheiro acabou } \\
(1=\text { não; } 2=\operatorname{sim})\end{array}$ \\
\hline Inclusão econômica (le) & $\begin{array}{l}\text { Grupamentos ocupacionais do trabalho principal do responsável pelo domicílio } \\
\text { (1=trabalhadores agrícolas; } 2=\text { ocupações mal definidas; } 3=\text { outros) (1) } \\
\text { Posição na ocupação principal do responsável pelo domicílio (1=sem } \\
\text { remuneração; } 2=\text { sem carteira assinada - outros empregos e trabalho doméstico, } \\
\text { produção e construção próprio consumo -; } 3=\text { militar; funcionário público } \\
\text { estatutário; conta própria e empregador; empregado com carteira assinada e } \\
\text { trabalhador doméstico com carteira assinada). } \\
\text { Número de horas trabalhadas semanalmente pelo responsável do domicílio na } \\
\text { semana de referência, numa escala que vai de } 1 \text { a } 98 \text { horas. }\end{array}$ \\
\hline
\end{tabular}

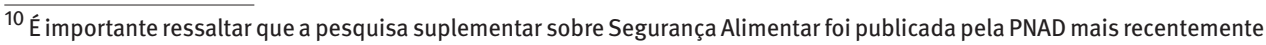
em 2009, fato que se mostrou relevante para esse estudo e que procura justificar a utilização da amostra do ano em referência.

${ }^{11}$ A autora propõe utilizar as seguintes categorias para medir as dimensões relacionadas à pobreza multidimensional: “i) conforto no lar (variável latente): existência de geladeira no domicílio (1=não; $2=\operatorname{sim}$ ); existência de televisão no domicílio ( $1=$ não, $2=\operatorname{sim}$ ); existência de banheiro (1=não, 2=sim); ii) saneamento básico (variável latente): abastecimento de água, origem (1=outra; $2=$ poço ou nascente; $3=$ =rede geral); abastecimento de água, canalização (1=não canalizada; $2=$ canalizada só na propriedade ou terreno; $3=$ canalizada em pelo menos um cômodo); iii) tipo de inserção no mercado de trabalho (variável latente): qualidade da ocupação / grau de garantias e proteção social (1=desocupado; 2=trabalhador não contribuinte; $3=$ trabalhador contribuinte); condição de inserção na organização social do trabalho (1=desinserido; $2=$ conta-própria; $3=$ empregado/trabalha para terceiros), incluindo as variáveis observadas: renda domiciliar per capita mensal (R\$); educação (anos completos de estudo )" (CODES, 2005, p.188), entre outras.
} 
(continuação)

\begin{tabular}{|c|c|}
\hline Variáveis latentes & Variáveis observáveis \\
\hline \multirow{3}{*}{$\begin{array}{l}\text { Inclusão por meio das } \\
\text { condições de moradia } \\
(\text { Icm) }\end{array}$} & $\begin{array}{l}\text { Domicílio possui banheiro ou sanitário } \\
(1=\text { não; } 2=\operatorname{sim})\end{array}$ \\
\hline & $\begin{array}{l}\text { Forma de escoadouro do banheiro ou sanitário } \\
\text { (1=fossa rudimentar; valas; direto para o rio, lago ou mar; } 2=\text { =fossa séptica não } \\
\text { ligada à rede coletora de esgoto ou pluvial; } 3=\text { rede coletora de esgoto ou pluvial e } \\
\text { fossa séptica ligada à rede coletora de esgoto ou pluvial) }\end{array}$ \\
\hline & $\begin{array}{l}\text { Destino do lixo domiciliar } \\
\text { (1=jogado em terreno baldio; jogado em rio; lago ou mar e outro destino; } \\
2=\text { =queimado ou enterrado na propriedade; } 3=\text { coletado direta ou indiretamente). }\end{array}$ \\
\hline
\end{tabular}

Fonte: IBGE. Pesquisa Nacional por Amostra de Domicílios (PNAD) 2009.

(1) Outras ocupações incluem: dirigentes em geral; profissionais das ciências e das artes; técnicos de nível médio; trabalhadores de serviços administrativos; trabalhadores dos serviços; vendedores e prestadores de serviços do comércio; trabalhadores da produção de bens e serviços e reparação e manutenção; membros das forças armadas e auxiliares.

\section{Análise dos resultados}

Verificou-se empiricamente que a pobreza possui características complexas, resultantes de uma inter-relação entre vários fatores que se manifestam na mesma sincronia e intensidades muito próximas, conforme pode ser comprovado pelos resultados apresentados nesta seção.

As análises subjacentes dos modelos mostraram que, no conjunto das formulações focalizadas na teoria, de maneira geral, as articulações dos fatores tendem a se aproximar do modelo proposto. No primeiro momento, constata-se que a confiabilidade das variáveis latentes que compõem o modelo hipotético sob a análise do Coeficiente de Crombach (a), ${ }^{12}$ em que valores superiores a 0,70 indicam que elas podem ser utilizadas na modelagem, foi observada para as três regiões metropolitanas estudadas.

Nessa perspectiva, analisa-se que a construção do modelo sinaliza uma boa estrutura teórica que, primeiramente, será estudada a partir da avaliação dos critérios de ajuste apresentados na Tabela 1. 0 RMSEA (Root Mean Square Error of Approximation) indica ajustamento ${ }^{13}$ global do modelo para grandes amostras, além de ser um dos mais indicados para modelagens que utilizam estruturas de covariâncias (CODES, 2005; SILVA, 2006). Neste estudo, foram empregados, como referência para esse índice com ajuste satisfatório, valores menores ou iguais a 0,08 (MACDONALD; RINGO HO, 2002; HAIR et al., 2009). Todos os modelos apresentaram bom ajuste, ao tomar como referência os índices de ajuste incremental CFI (Comparative Fit Index), que possui em sua estrutura muitas propriedades satisfatórias, tais como seu alto poder de medir a sensibilidade relativa no ajuste, e o TLI (Índice de Tucker-Lewis). Ambos índices de ajuste incremental (CFI e TLI) têm seus valores

\footnotetext{
${ }^{12}$ Codes (2005, p.179) menciona que "quando se trata de estimar a confiabilidade de um fator, que é composto por um grupo de variáveis observáveis, o cálculo é feito com base nas correlações entre elas. A medida mais comum de confiabilidade é o Coeficiente Alfa de Cronbach (a), que afere a consistência interna entre aqueles itens que compõem o fator. De modo simplificado, tal coeficiente fornece a proporção da variação da variável latente que é comum entre os itens que a compõem. Em outras palavras, ele indica a proporção da informação social 'verdadeira' ou 'confiável' existente naquele fator. Em geral, variáveis latentes que tenham valores de a iguais ou superiores a 0,70 são consideradas satisfatórias. A partir desse patamar, as variáveis latentes são vistas como suficientemente confiáveis para participarem das modelagens".

13 O RMSEA é também conhecido como índice de ajuste parcimonioso e foi elaborado por Stiger e Lind (1980).
} 
variando de 0 a 1 , sendo que quanto mais próximo de 1 melhor é o ajuste. Diante dos valores expostos na Tabela 1, é possível verificar que todos os modelos propostos, tanto de primeira quanto de segunda ordem, apresentaram bons ajustes, ou seja, os dados empíricos sinalizam estar representando de forma satisfatória a teoria estudada.

TABELA 1

Índices de ajuste para os modelos de Análise Confirmatória (AFC) de primeira e segunda ordens Regiões Metropolitanas de Fortaleza, Recife e Salvador - 2009

\begin{tabular}{lccccccc}
\hline \multirow{2}{*}{$\begin{array}{c}\text { Regiões } \\
\text { metropolitanas }\end{array}$} & \multicolumn{3}{c}{ AFC - primeira ordem } & & \multicolumn{3}{c}{ AFC - segunda ordem } \\
\cline { 2 - 3 } & RAMSEA & CFI & TLI & & RAMSEA & CFI & TLI \\
\hline Fortaleza & 0,074 & 0,972 & 0,964 & & 0,074 & 0,972 & 0,964 \\
Recife & 0,067 & 0,963 & 0,953 & & 0,065 & 0,965 & 0,956 \\
Salvador & 0,068 & 0,942 & 0,927 & & 0,066 & 0,944 & 0,931 \\
\hline
\end{tabular}

Fonte: IBGE. Pesquisa Nacional por Amostra de Domicílios (PNAD) 2009

Nota: RAMSEA - Root Mean Square Error of Approximation; CFI - Comparative Fit Index; TLI - Índice de Tucker-Lewis.

As cargas fatoriais, que mostram a relação de cada variável mensurável com a respectiva dimensão da pobreza, são apresentadas na Tabela 2. Verifica-se, por exemplo, que na Região Metropolitana de Recife a aquisição do bem material "carro ou motocicleta"14 está relacionada com o aumento do bem-estar econômico do chefe do domicílio em torno de $77 \%$, sendo que as outras duas regiões metropolitanas apresentam cenário parecido. Percebe-se que nas três regiões estudadas o aumento do bem-estar econômico está associado ao aumento das variáveis observadas: renda, TV, geladeira, celular e carro/ motocicleta. Nesse caso, renda e consumo despontam como materialização da redistribuição dos recursos materiais e financeiros disponíveis na economia, destacando-se como variáveis preponderantes para analisar a magnitude da satisfação bem-estar econômico do indivíduo (ROCHA, 1997, 1998, 2005; BARROS; FERES, 1998; BARROS; MENDONÇA, 1995; NERI, 2008, 2011; NERI; SOARES, 2010).

Ao analisar a dimensão capacidade, verifica-se que nas três regiões metropolitanas foi possível constatar forte presença de algum tipo de insegurança ${ }^{15}$ alimentar na população-alvo do estudo. Se for considerada uma média da variância para todas variáveis que registram a insegurança alimentar nas regiões analisadas, chega-se a um valor negativo acima de 0,90 , sinalizando que o aumento na capacidade só será alcançado se a insegurança alimentar for reduzida. Tal fato indica a fragilidade que essas condições desfavoráveis podem acarretar para a saúde da população, principalmente quando se verifica que essa deficiência nutricional tende a prejudicar principalmente as crianças

\footnotetext{
$\overline{14}$ A demanda por "carro/motocicleta" está em trajetória crescente no país desde o final de 2008 com a facilidade do crédito, aumentando, nesse momento, o nível de confiança dos consumidores em relação ao comprometimento de parte da sua renda (SILVA; MORAES, 2012, p. 3).

${ }^{15}$ Segundo Nota Metodológica da PNAD 2009: “Insegurança alimentar está diretamente relacionada com a incapacidade ou incerteza de se obter o alimento com qualidade e quantidade suficientes, por meios socialmente aceitos. Está relacionada com escassez de alimentos, falta de acesso aos alimentos, desnutrição e pobreza e, também se expressa pelo medo ou preocupação da pessoa com a possibilidade de vir a ter fome” (IBGE,2009)
} 
em idade escolar (MACHADO, 2007). A dimensão capacidade, no modelo proposto, aumenta a educação.

TABELA 2

Cargas fatoriais das variáveis observadas em suas correspondentes variáveis latentes no modelo de primeira ordem

Regiões Metropolitanas de Fortaleza, Recife e Salvador - 2009

\begin{tabular}{|c|c|c|c|c|c|c|c|c|c|c|c|c|}
\hline \multirow{2}{*}{$\begin{array}{l}\text { Variáveis } \\
\text { observadas }\end{array}$} & \multicolumn{4}{|c|}{ RM de Fortaleza } & \multicolumn{4}{|c|}{ RM de Recife } & \multicolumn{4}{|c|}{ RM de Salvador } \\
\hline & Bee & Capac & le & $\mathrm{Icm}$ & Bee & Capac & le & $\mathrm{Icm}$ & Bee & Capac & le & $\mathrm{Icm}$ \\
\hline Renda & 0,797 & & & & 0,765 & & & & 0,847 & & & \\
\hline TV & 0,580 & & & & 0,571 & & & & 0,575 & & & \\
\hline Geladeira & 0,609 & & & & 0,582 & & & & 0,654 & & & \\
\hline Celular & 0,632 & & & & 0,560 & & & & 0,447 & & & \\
\hline $\begin{array}{l}\text { Carro/ } \\
\text { motocicleta }\end{array}$ & 0,726 & & & & 0,770 & & & & 0,567 & & & \\
\hline Educação & & 0,548 & & & & 0,536 & & & & 0,558 & & \\
\hline $\begin{array}{l}\text { Alimentos } \\
\text { acabar }\end{array}$ & & $-0,947$ & & & & $-0,945$ & & & & $-0,878$ & & \\
\hline $\begin{array}{l}\text { Alimentos } \\
\text { acabar antes }\end{array}$ & & $-0,996$ & & & & $-0,964$ & & & & $-0,964$ & & \\
\hline $\begin{array}{l}\text { Comeram } \\
\text { alguns } \\
\text { alimentos }\end{array}$ & & $-0,942$ & & & & $-0,955$ & & & & $-0,938$ & & \\
\hline $\begin{array}{l}\text { Grupo } \\
\text { ocupacional }\end{array}$ & & & 0,752 & & & & 0,723 & & & & 0,408 & \\
\hline $\begin{array}{l}\text { Posiçãão na } \\
\text { ocupação }\end{array}$ & & & 0,532 & & & & 0,513 & & & & 0,456 & \\
\hline $\begin{array}{l}\text { № de horas } \\
\text { trabalhadas }\end{array}$ & & & - & & & & 0,615 & & & & 0,677 & \\
\hline Banheiro & & & & 0,782 & & & & 0,260 & & & & 0,611 \\
\hline $\begin{array}{l}\text { Escoamento } \\
\text { sanitário }\end{array}$ & & & & 0,417 & & & & 0,667 & & & & 0,415 \\
\hline Lixo & & & & 0,598 & & & & 0,432 & & & & 0,310 \\
\hline
\end{tabular}

Fonte: IBGE. Pesquisa Nacional por Amostra de Domicílios (PNAD) 2009.

Nota: Bee - bem-estar econômico; Capac - capacidade; le - inclusão econômica; Icm - inclusão por meio das condições de moradia.

No quesito mercado de trabalho dos chefes dos domicílios, foi na RM de Salvador que as variáveis mensuradas referentes à posição na ocupação e grupo de ocupação apresentaram as menores cargas fatoriais, na dimensão inclusão econômica. Vale salientar algumas características sobre o mercado de trabalho nesta região, de acordo com os dados da PNAD 2009. No que tange ao construto inclusão econômica, a tendência dos resultados foi muito similar à encontrada para a RM de Recife, ou seja, o "número de horas trabalhadas" registrou o maior impacto nesta dimensão $(0,677)$, seguido por "posição na ocupação do trabalho principal" $(0,456)$ e "grupo de ocupação no trabalho principal da ordem" $(0,403)$. Em termos comparativos, tanto a RM de Salvador como a de Recife registraram chefes de domicílios trabalhando com carteira assinada em proporções maiores do que a encontrada na RM de Fortaleza: 44,10\%, 43,62\% e 38,14\%, respectivamente. Na posição diametralmente oposta, os indivíduos que trabalhavam sem 
carteira assinada correspondiam a 16,42\% na RM de Salvador, 16,97\% na de Recife e $22,22 \%$ na de Fortaleza, ou seja, o mercado de trabalho nesta última região se mostrou mais frágil do que nas outras duas.

A Tabela 3 traz a intensidade das intercorrelações entre as dimensões de primeira ordem: bem-estar econômico; capacidade; inclusão econômica; e inclusão por meio das condições de moradia. Constata-se que, para a RM de Fortaleza, as correlações entre todas as dimensões se mostram relativamente altas. Nas RMs de Recife e Salvador, o construto inclusão econômica apresentou a menor intensidade na correlação com as outras dimensões que compõem o modelo de primeira ordem. Diante desse fato, são necessários alguns posicionamentos dessa dimensão - inclusão econômica - com as outras - bem-estar econômico, capacidade e inclusão por meio das condições de moradia.

TABELA 3

Correlação entre fatores latentes no modelo de primeira ordem Regiões Metropolitanas de Fortaleza, Recife e Salvador - 2009

\begin{tabular}{|c|c|c|c|c|c|c|c|c|c|c|c|c|}
\hline \multirow{2}{*}{$\begin{array}{l}\text { Variáveis } \\
\text { latentes }\end{array}$} & \multicolumn{4}{|c|}{ RM de Fortaleza } & \multicolumn{4}{|c|}{ RM de Recife } & \multicolumn{4}{|c|}{ RM de Salvador } \\
\hline & Bee & Capac & le & $\mathrm{Icm}$ & Bee & Capac & le & $\mathrm{Icm}$ & Bee & Capac & le & $\mathrm{Icm}$ \\
\hline Bee & 1 & & & & 1 & & & & 1 & & & \\
\hline Capac & 0,669 & 1 & & & 0,696 & 1 & & & 0,717 & 1 & & \\
\hline le & 0,598 & 0,445 & 1 & & 0,345 & 0,194 & 1 & & 0,207 & 0,183 & 1 & \\
\hline $\mathrm{Icm}$ & 0,668 & 0,497 & 0,892 & 1 & 0,592 & 0,469 & 0,166 & 1 & 0,749 & 0,546 & 0,145 & 1 \\
\hline
\end{tabular}

Fonte: IBGE. Pesquisa Nacional por Amostra de Domicílios (PNAD) 2009.

Nota: -Bee - bem-estar econômico; Capac - capacidade; le - inclusão econômica; Icm - inclusão por meio das condições de moradia.

No que se refere ao bem-estar econômico e à capacidade, fez-se uma busca nos dados para entender o porquê desse comportamento da dimensão inclusão econômica nas RMs de Recife e Salvador. Primeiramente, como o bem-estar econômico na RM de Recife tem seu crescimento em maior grandeza atribuído à "proxy" acesso ao crédito - carro/motocicleta -, é importante mencionar o trabalho de Galeano e Feijó (2011). Nele, as autoras afirmam que existe forte indicativo de que a maior disponibilidade de crédito e financiamento está diretamente ligada a maiores taxas de crescimento econômico. Essa linha de raciocínio pode ser sustentada pela análise do crédito disponível para o consumo por meio do saldo de operações de crédito do sistema financeiro nacional para pessoa física, em 2009, sendo possível constatar que o Estado do Ceará demandou aproximadamente R\$99.051 milhões, Pernambuco R\$127.459 milhões e Bahia R\$201.682 milhões desses recursos. ${ }^{16}$ Se compararmos o crescimento econômico desses Estados, verifica-se que o Ceará cresceu menos (9,33\%) do que Pernambuco (11,34\%) e Bahia (12,81\%). Assim, é possível observar a existência de correlação entre crescimento econômico e disponibilidade de crédito para pessoas físicas, conforme defendido pelas autoras. Sob esse prisma, é justificável perceber que o consumo de bens nesse período (2008-2009) ficou mais atrelado ao acesso ao crédito do que propriamente à inclusão econômica.

\footnotetext{
$\overline{16}$ Segundo o Banco Central do Brasil. Disponível em: 〈www.bacen.gov.br〉.
} 
Seguindo essa ótica, verifica-se que a inclusão econômica na atividade econômica na RM de Recife tende a estar fortemente ligada ao crescimento econômico desse período. Nesse sentido, o crescimento econômico passa a ser o determinante na alocação de mão de obra, o chamado efeito multiplicador ${ }^{17}$ de Keynes (1992). E a mesma visão pode ser estendida para a RM de Salvador, pois se trata da região do Nordeste que mais recebe investimentos estruturadores, tanto os já instalados quanto os novos, como, por exemplo, o polo petroquímico de Camaçari, a montadora Ford Motors do Brasil e a fábrica de matéria-prima para herbicida Monsanto. Esses investimentos se tornaram um dos principais propulsores da expansão e diversificação da base produtiva do Estado da Bahia e principalmente da RM de Salvador.

No que se refere à ocupação dos espaços urbanos, a RM de Salvador apresenta certa similaridade com a de Recife e, por isso, um aumento em infraestrutura em bairros mais centrais implicará também melhorias nas condições de moradia da população menos favorecida, pois parte dessas moradias está localizada nesses bairros ricos/nobres, ou seja, é possível visualizar em Salvador ou Recife moradias de alto padrão muito próximas de moradias bem precárias/favelas.

De acordo com a Tabela 4, a dimensão de ordem superior - nível de pobreza - na RM de Fortaleza explica a variância das dimensões de nível inferior: bem-estar econômico com $88,5 \%$; capacidade com $49,6 \%$; inclusão econômica com $46,8 \%$; e inclusão por meio das condições de moradia com 55,3\%. Na RM de Recife verifica-se que o bem-estar econômico tem sua variância explicada pela dimensão superior em 92,4\%, a capacidade em torno de 52,40\%, a inclusão econômica aproximadamente $9,20 \%$ e a inclusão por meio das condições de moradia em $66,40 \%$. Na RM de Salvador esses valores são de $71,23 \%, 52,85 \%, 5,20 \%$ e $51,84 \%$, respectivamente.

TABELA 4

Correlação do construto de segunda ordem (pobreza) com os construtos do modelo de primeira ordem Regiões Metropolitanas de Fortaleza, Recife e Salvador - 2009

\begin{tabular}{|c|c|c|c|}
\hline \multirow{2}{*}{$\begin{array}{l}\text { Variáveis } \\
\text { latentes }\end{array}$} & \multicolumn{3}{|c|}{ Pobreza } \\
\hline & RM de Fortaleza & RM de Recife & RM de Salvador \\
\hline Bee & 0,941 & 0,961 & 0,988 \\
\hline Capac & 0,704 & 0,724 & 0,727 \\
\hline le & 0,684 & 0,304 & 0,228 \\
\hline $\mathrm{Icm}$ & 0,744 & 0,815 & 0,720 \\
\hline
\end{tabular}

Fonte: Pesquisa Nacional por Amostra de Domicílios (PNAD) 2009.

Nota: Bee - bem-estar econômico; Capac - capacidade; le - inclusão econômica; Icm - inclusão por meio das condições de moradia.

\footnotetext{
17 "Matematicamente, a expressão é dada da seguinte maneira: $\left.\Delta Y_{w}\right\rangle \Delta C_{W}$, onde $Y_{w}$ representa a renda em unidades de salário e $C_{W}$ denota o consumo imediato em unidades de salário. Assim, a propensão marginal a consumir é dada pela expressão $\mathrm{d} C_{w} / \mathrm{d} Y_{W}$ e sinaliza como se dividirá o próximo incremento da produção entre o consumo e o investimento. Isso porque $\Delta Y_{w}=\Delta C_{W}+\Delta I_{w}$, onde $\Delta C_{w}$ e $\Delta I_{w}$ são incrementos do consumo e do investimento, de maneira que podemos escrever $\Delta Y_{w}=k \Delta I_{w}$, onde 1-1/ké igual à propensão marginal a consumir. $E$ krepresenta o multiplicador de investimento, o mesmo indica que, quando se produz um acréscimo no investimento agregado, a renda sobe num montante igual a $k$ vezes o acréscimo do investimento" (KEYNES; 1992, p. 101).
} 
No que tange ao construto inclusão econômica, a baixa correlação em relação às outras dimensões no modelo de primeira ordem - nível inferior - das RMs de Recife e Salvador foi captada pela dimensão de segunda ordem - pobreza. Essa linha de raciocínio pode ser sustentada a partir do momento em que se analisa a baixa intensidade na correlação da dimensão inclusão econômica em relação a bem-estar econômico, capacidade e inclusão por meio das condições de moradia.

Diante do que foi apresentado, é possível verificar que a dimensão pobreza - modelo de segunda ordem - demonstra a multidimensionalidade das características do fenômeno da pobreza por meio da forte correlação com bem-estar econômico, capacidade, inclusão econômica (exceto nas RMs de Recife e Salvador que apresentaram baixa correlação) e inclusão por meio das condições de moradia.

\section{Considerações finais}

Percebe-se, ao longo das análises, que em todas as regiões metropolitanas estudadas 0 aumento do bem-estar econômico está associado ao aumento principalmente das variáveis renda e consumo. E este construto tem forte impacto imediato na pobreza. Tal fato faz a privação econômica se tornar um dos instrumentos de análise para identificar o fenômeno da pobreza (ROCHA, 2005). No entanto, a análise do modelo de segunda ordem demonstra a multidimensionalidade das características do fenômeno da pobreza por meio da forte correlação com bem-estar econômico, capacidade, inclusão econômica (exceto nas RMs de Recife e Salvador) e inclusão por meio das condições de moradia.

Na perspectiva exposta, a estrutura teórica - a pobreza - se depara com uma realidade complexa. Somente por meio de ações e execuções de políticas públicas mais amplas que venham focalizar as outras dimensões de forma eficiente é que se poderá realmente promover a redução da pobreza de grande parcela da sociedade. Em síntese, este artigo mostra uma medição quantitativa das múltiplas facetas do fenômeno da pobreza e sinaliza o caminho a ser percorrido para o enfrentamento desse estágio de privação, procurando colaborar com as propostas metodológicas que fazem uso da PNAD para o estudo multidimensional da pobreza, sob a ótica de algumas dimensões, como a geração de emprego e a ampliação do acesso a serviços públicos e moradia.

\section{Referências}

ALKIRE, S. The capability approach and well-being measurement for public policy. Oxford Poverty \& Human Development Initiative (OPHI), March 2015 (Working paper, n. 94).

ASPAROUHOV, T.; MUTHÉN, B. Resampling methods in Mplus for complex survey data. 2010. Disponível em: 〈www.statmodel.com/download/Resampling_Methods5.pdf〉. Acesso em: 17 ago. 2015.

BARBOSA, A. B.; GAVIOLI, A. P. T.; YAMANISHI, S. P. Condições de moradia da população de baixa renda: o caso do Jardim Petrópolis. Revista de Ciências Humanas da Unipar, v. 11, n. 3, 2003. 
BARRETO, M. L. et al. Sucessos e fracassos no controle de doenças infecciosas no Brasil: o contexto social e ambiental, políticas, intervenções e necessidades de pesquisa. The Lancet, 9 de maio de 2011. Disponivel em: 〈http://download.thelancet.com/flatcontentassets/pdfs/ brazil/brazilpor3.pdf〉. Acesso em: 11 jun. 2012.

BARROS, R. de P.; FERES, J. C. A proposal for drawing up indigence lines. In: SEGUNDO ENCONTRO DO EXPERT GROUP ON POVERTY STATISTICS. Rio de Janeiro, maio de 1998.

BARROS, R. P.; MENDONÇA, R. A evolução do bem-estar, pobreza e desigualdades no Brasil ao longo das três últimas décadas - 1960-1980. Pesq. Plan. Econ., v. 25, n. 1, p. 115-164, 1995.

BARROS, R. P.; CARVALHO, M.; FRANCO, S. Pobreza multidimensional no Brasil. Rio de Janeiro: Ipea, 2006 (Texto para discussão, n. 1227).

BETARRELLI JR., A. A. Custo de acessibilidade entre residências e trabalho: um enfoque das características individuais, familiares e locais. Belo Horizonte: UFMG/Cedeplar, 2010 (Texto para discussão, n. 407).

BETTI, G.; D’AGOSTINO, A.; NERI, L. Panel regression models for measuring multidimensional poverty dynamics. Statistical Methods and Applications, v. 11, n. 3, p. 359-369, 2002.

BOLLEN, K. A. Structural equation models with latent variables. New York: John Wiley \& Sons, 1989.

BOURGUIGNON, F.; CHAKRAVARTY, S. The measurement of mutidimensional poverty. Journal of Economic Inequality, n. 1, p. 25-49, 2003.

BROWN, T. A. Confirmatory factor analysis for applied research. New York: The Guilford Press, 2006.

BURCHARDT, T.; LE GRAND, J.; PIACHAUD, D. Degress of exclusion: developing a dynamic, multidimensional measure. In: HILLS, J.; LE GRAND, J.; PIACHAUD, D. (Eds.). Understanding social exclusion. New York: Oxford University Press, 2002. p. 30-43.

BYRNE, B. M. Structural equation modeling with lisrel, prelis, and simplis: basic concepts, applications and programming. USA: Lawrence Erlbaum Associates, 1998.

CITRO, C. F.; MICHAEL, R. T. Measuring poverty. A new approach. Washington: National Academy Press, 1995.

CODES, A. L. M. Modelagem de equações estruturais: uma contribuição metodológica para o estudo da pobreza. 2005. 207f. Tese (Doutorado em Ciências Sociais) - Universidade Federal da Bahia, Salvador, 2005.

COLASANTO, D.; KAPTEYN, A.; VAN DER GAAG, J. Two subjective definitions of poverty: results from the Wisconsin Basic Needs Study. The Journal of Human Resources, v. 19, n. 1, p. 127-138, 1984.

CORRÊA, L. F. C; LIMA, P. R. Impacto redistributivo dos gastos sociais nas regiões metropolitanas no Nordeste do Brasil em 2004. Análise Econômica, ano 29, n. 55, p. 309-332, mar. 2011.

DEUTSCH, J.; SILBER, J. Measuring multidimensional poverty: an empirical comparison of various approaches. Review of Income and Weath, series 51, n. 1, 2005.

DIALLO, F. L. Analysing multidimensional poverty in Guinea using the fuzzy set approach. Dakar, Senegal: Cheikh Anta Diop University of Dakar (UCAD), Senegal Consortium for Social and Economic Research (CRES), 2010.

GALEANO, E. V.; FEIJÓ, C. Crédito e crescimento econômico: evidências a partir de um painel de dados regionais para a economia brasileira nos anos 2000. Niterói: Universidade Federal Fluminense, 2011 (Texto para discussão n. 32).

HAIR, J. F. et al. Análise multivariada de dados. 6. ed. São Paulo: Bookman, 2009. 
HOFFMANN, R. Pobreza, insegurança alimentar e desnutrição no Brasil. Estudos Avançados, v. 9, n. 24, maio/ago. 1995.

HOLANDA, M. C. et al. Inclusão social no Ceará: uma proposta metodológica. Fortaleza: Ipece, 2003 (Texto para discussão, n. 4).

IBGE - Instituto Brasileiro de Geografia e Estatística. Microdados 2009. Disponível em: 〈http:// www.ibge.gov.brr. Acesso em: 20 mar. 2012.

KAGEYAMA, A.; HOFFMANN, R. Pobreza no Brasil: uma perspectiva multidimensional. Economia e Sociedade, v. 15, n. 1 (26), p. 79-112, jan./jun. 2006.

KEYNES, J. M. A teoria geral do emprego, do juro e da moeda. Tradução de Mário R. Cruz. São Paulo: Atlas, 1992 (Coleção Os Economistas).

LAMARE, S. L. D. Modelagem de equações estruturais na melhoria da gestão. In: ENCONTRO NACIONAL DE ENGENHARIA DE PRODUÇÃO, 22. Anais... Curitiba: Abepro, 2002.

LISTER, R. Poverty. Cambridge: Blackwell/Polity Press, 2004.

MACHADO, D. C. Efeitos da saúde na idade de entrada à escola. Niterói: Universidade Federal Fluminense, 2007 (Texto para discussão, n. 221).

MARIÓ, E. G. Conclusões e recomendações. In: MARIÓ, E. G.; WOOLCOK, M. (Orgs.). Exclusão social e mobilidade no Brasil. Brasília: Ipea, 2005. p. 133-145.

MCDONALD, R. P.; RINGO HO, M.-H. Principles and practice in reporting structural equation analyses. Psychological Methods, v. 7, n. 1, p. 64-82, 2002.

MUTHÉN, L. K.; MUTHÉN, B. O. Mplus: statistical analysis with latent variables. User's guide. (1998-2007). Fifth Edition. Los Angeles: Muthén \& Muthén, Nov. 2007.

NERI, M. Desigualdade de renda na década. Rio de Janeiro: FGV/CPS, 2011. Disponível em: 〈http://www.cps.fgv.br/cps/bd/DD/DD_Neri_Fgv_TextoFim3.pdf〉. Acesso em: 18 maio 2011.

A Geografia das fontes de renda. Rio de Janeiro: FGV, 2008. Disponível em: 〈http:// www.fgv.br/cps/docs/geofonte.pdf〉. Acesso em: 11 maio 2011.

NERI, M. C.; SOARES, W. L. Pobreza, ativos e saúde no Brasil. Rio de Janeiro: FGV, EPGE, 2010 (Ensaios Econômicos, 465).

NERY, T. C. S. Saneamento: ação de inclusão social. Estudos Avançados, v. 18, n. 50, p. 313-321, 2004.

OPEL, A. E. A. The social content of labor markets in Dhaka Slums. International Journal of Development, v. 12, p. 735-50, 2000.

ORSHANSK, M. Counting the poor: another look at the poverty profile. Social Security Bulletin, v. 28, n. 1, p. 3-29, 1965.

RAMOS, L.; REIS, J. G. Salário mínimo, distribuição de renda e pobreza no Brasil. Pesquisa de Planejamento Econômico, v. 25, n. 1, p. 99-114, 1995.

RAVALLION, M. Issues in measuring and modeling poverty. Washington: World Bank, Poverty and Human Resources Division, Policy Research Department, 1996 (Policy Research Working Paper, 1615).

REIS, C. N.; DALAGASPERIANA, E. C. Complexidade conceitual de pobreza: notas sobre diferentes abordagens conceituais. In: ENCONTRO NACIONAL DE ECONOMIA POLÍTICA, 14. Anais... São Paulo: Sociedade Brasileira de Economia Política, 2009.

RINGEN, S. The possibility of politics. A study in the political economy of the Welfare State. Oxford: Clarendon Press, 1987. 
On multidimensional indices of poverty. Washington: World Bank, Poverty and Human Resources Division, Policy Research Department \# 5580, 2011.

ROCHA, S. Pobreza no Brasil. Afinal, de que se trata? Rio de Janeiro: FGV, 2005.

Pobreza e indigência no Brasil: algumas evidências empíricas com base na PNAD 2004. Nova Economia, v. 16, n. 2, p. 265-299, maio/ago. 2006.

Estabelecimento e comparação de linha de pobreza no Brasil. Rio de Janeiro: Ipea, 1998 (Texto para discussão, n. 153).

Do consumo observado à linha de pobreza. Pesquisa de Planejamento Econômico, v. 27, n. 2, p. 313-352, 1997.

RODRIGUES, R. I. O lugar dos pobres e a violência na cidade: um estudo para o município de São Paulo, 2005. Disponivel em: 〈www.anpec.org.br/encontro2005/artigos/A05A154.pdf〉. Acesso em: 12 jun. 2012.

SCALON, M. C. Mobilidade social no Brasil: padrões e tendências. Rio de Janeiro: Revan, IUPERJUCAM, 1999.

SEN, A. K. A idéia de justiça. Tradução de Denise Bottamann e Ricardo Doninelli Mendes. São Paulo: Companhia das Letras, 2011.

Social exclusion: concept, application, and scrutiny. Manila, Philippines: Asian Development Bank, 2000 (Social development papers, n. 1).

Development as freedom. New York: Alfred A. Knoff, 1999.

SILVA, J. S. F. Modelagem de equações estruturais: apresentação de uma metodologia. 2006. 105f. Dissertação (Mestrado em Engenharia de Produção) - Universidade Federal do Rio Grande do Sul, Porto Alegre, 2006.

SILVA, T. L. F.; MORAES, G. I. Fatores macroeconômicos que influenciaram o crédito pessoa física: recursos livres, no período de 2004-2011. In: ENCONTRO DE ECONOMIA GAÚCHA, 6. Anais... Porto Alegre: PUC-RS, 2012.

SINGER, P. Desemprego e exclusão social. Revista São Paulo em Perspectiva, v. 10, n.1, 1996.

SUMNER, A. Economic well-being and non-economic well-being. A review of the meaning and measurement of poverty. Helsinki: UNU World Institute for Development Economics Research (UNU-WIDER), 2004.

TEJADA, C. A. O.; JACINTO, P. A.; SANTOS, A. M. A. Pobreza e saúde: evidências de causalidade em um painel de dados para o Brasil. In: ENCONTRO REGIONAL DE ECONOMIA - ANPEC/SUL, 11. Anais... Curitiba: UFPR, 2008.

VALLE FURTADO, C. S. Classificação dos pobres: questões, construção e análise. Sociologias, v. 13, n. 26, p. 306-330, jan./abr. 2011.

VAZ, F. M.; SOARES, S. S. D. Linhas de pobreza subjetivas para o Brasil. In: ENCONTRO NACIONAL DE ECONOMIA, 36. Anais...Salvador: Anpec, 2008.

VIDIGAL, C. B. R. Índice de bem-estar econômico: uma proposta para os estados brasileiros. 2011. 123f. Dissertação (Mestrado em Economia Aplicada) - Escola Superior de Agricultura "Luiz de Queiroz", Piracicaba, 2011.

WAGLE, U. R. Multidimensional poverty measurement: concepts and applications. New York: Springer, 2008.

- Volver a pensar la pobreza: definición y mediciones. Revista Internacional de Ciências Sociales, n. 171, 2002. Disponível em: 〈http://www.oei.es/salactsi/wagle.pdf〉. Acesso em: 24 jul. 2015. 


\title{
Sobre os autores
}

Lucilena Ferraz Castanheira Corrêa é doutora em Economia pelo Programa de Pós-Graduação em Economia (Pimes) da Universidade Federal de Pernambuco (UFPE). Professora adjunta da Universidade Federal de Pernambuco (UFPE) - campus Agreste.

João Policarpo Rodrigues Lima é PhD em Economia pela University College London. Professor titular da Universidade Federal de Pernambuco (UFPE).

Luís Henrique Romani de Campos é doutor em Economia pelo Programa de Pós-Graduação em Economia (Pimes) da Universidade Federal de Pernambuco (UFPE). Pesquisador titular e diretor de Pesquisas Sociais da Fundação Joaquim Nabuco.

\section{Endereço para correspondência}

\author{
Lucilena Ferraz Castanheira Corrêa \\ Rua Frei Leandro, 70 \\ 51011-600 - Recife-PE, Brasil
}

\begin{abstract}
An analysis of multidimensional poverty in metropolitan areas of Brazil's Northeast

Poverty is a highly complex social phenomenon and the problematic of this stage of deprivation is formed by the aggregation of various social factors. As such, the more information incorporated into the study involving this stage of deprivation, the more accurate the diagnosis of reality. To do so, in this paper an analytical tool used is the method of Structural Equation Modeling (SEM), utilizing microdata from the National Household Sample Survey (PNAD) of 2009, and focusing on the metropolitan areas of the Brazilian Northeast: Fortaleza, Recife and Salvador. Four dimensions of poverty were defined: capacity, economic welfare, economic inclusion and inclusion through housing conditions, for which a correlation analysis will be performed. Next, these dimensions are analyzed from the perspective of their impact over poverty. The results of this study reinforce the multidimensional features of this state of social and economic deprivation, as indicated in other research, but here based on modeling better suited to the theoretical approach.
\end{abstract}

Keywords: Poverty. Economic welfare. Capability. Economic inclusion. Inclusion through housing conditions.

\section{Resumen}

Un análisis de la pobreza multidimensional del Nordeste metropolitano con el uso del modelo de ecuaciones estructurales

La pobreza es un fenómeno social muy complejo y la problemática de ese estadio de privación se constituye por la agregación de diversos factores sociales. Desde esta perspectiva, cuanta más información se incorpora a los estudios que abordan este estadio de privación, más preciso será el diagnóstico de esta realidad. Para ello, se utiliza como herramienta de análisis el método del modelo de ecuaciones estructurales (SEM por su denominación en inglés), empleando datos de la Pesquisa Nacional por Amostra de Domicílios (PNAD) de 2009, y se focaliza el análisis en las regiones metropolitanas del Nordeste: Fortaleza, Recife y Salvador. Se definen cuatro 
dimensiones de la pobreza a partir de las que se realiza el análisis de correlación: la capacidad, el bienestar económico, la inclusión económica y la inclusión mediante las condiciones de vivienda, y en un segundo momento estas dimensiones se analizan bajo la óptica de su impacto sobre la pobreza. Los resultados de este trabajo refuerzan la existencia multidimensional de las características de este estadio de privación social y económica, ya señalada por otros estudios, pero se avanza a partir de la aplicación un modelo más adecuado al andamiaje teórico sobre el tema.

Palabras clave: Pobreza. Bienestar económico. Capacidad. Inclusión económica. Inclusión mediante las condiciones de vivienda.

Recebido para publicação em 25/04/2015

Recomendado para publicação 19/07/2015

Aceito para publicação em 25/08/2015 
